\title{
Subdivide the Shape of Numbers and a Theorem of Ring
}

\author{
Ji Peng \\ Department of Electronic Information, Nanjing University, Nanjing, China \\ Email:mcfroo@sina.com
}

How to cite this paper: Peng, J. (2020) Subdivide the Shape of Numbers and a Theorem of Ring. Open Access Library Journal, 7: e6719.

https://doi.org/10.4236/oalib.1106719

Received: August 17, 2020

Accepted: September 19, 2020

Published: September 22, 2020

Copyright $\odot 2020$ by author(s) and Open Access Library Inc.

This work is licensed under the Creative Commons Attribution International License (CC BY 4.0).

http://creativecommons.org/licenses/by/4.0/

\begin{abstract}
This article is based on the concept of Shape of numbers, introduce more shapes, obtain the calculation formulas and find an explanation of the formulas. By observing and associating, show a law about the symmetry of Ring.
\end{abstract}

\section{Subject Areas \\ Discrete Mathematics}

\section{Keywords}

Shape of Numbers, Calculation Formula, Symmetry, Combinatorics, Ring

\section{Introduction}

Peng, J. introduced the definition of Shape of numbers in [1]:

$$
\left(K_{1}, K_{2}, \cdots, K_{M}\right), K_{i} \in N, K_{1}<K_{2}<\cdots<K_{M}
$$

there are $M-1$ intervals between adjacent numbers. Use $A$ for continuity and $B$ for discontinuity, record as a string of $M-1$ characters (e.g. $A A B B \ldots$ ) to represents a catalog.

Define collection of a catalog as Shape of numbers. Use the symbol $P X$ to represent a catelog (if $M=1$ then $P X=1$ ).

The single $\left(K_{1}, K_{2}, \cdots, K_{M}\right)$ is an Item, $K_{1} K_{2} \cdots K_{M}$ is the product of an item.

For example:

$$
\begin{gathered}
(1,2,4),(1,2,8),(2,3,6) \in P X=A B,(1,3,5),(1,3,6),(2,4,6) \in P X=B B, \\
(1,2,5,6),(2,3,6,7),(2,3,7,8),(1002,1003,6789,6790) \in P X=A B A \\
P M(P X)=\text { Count of numbers of } P X, P A(P X)=\text { Count of } A, P B(P X)
\end{gathered}
$$


$=$ Count of $B$

$$
\rightarrow P M(P X)=P A(P X)+P B(P X)+1
$$

$|P X|=$ Count of items belonging to $P X$

$M I N(P X)=$ Minimum product of $P X: M I N(A A)=1 \times 2 \times 3$,

$\operatorname{MIN}(A B)=1 \times 2 \times 4$

$I D X(P X)=2+P A(P X)+2 \times P B(P X)=P M(P X)+P B(P X)+1:$

$\operatorname{IDX}(A A)=4, \quad I D X(A B)=5$

$\operatorname{SUM}(N, P X)=$ Sum of all product of items belonging to $P X$ in $[1, N-1]$

For example: $\operatorname{SUM}(6, A B)=1 \times 2 \times 4+1 \times 2 \times 5+2 \times 3 \times 5$

$\operatorname{END}(N, P X)=$ Set of items belonging to $P X$ with the maximum factor $=$ $N-1$

For example: $\operatorname{END}(6, B)=\{(1,5),(2,5),(3,5)\}$

[1] obtain the conclusion:

$$
\begin{gathered}
\mid \text { Items in } S U M(N, P X) \mid=\left(\begin{array}{c}
N-P M(P X) \\
P B(P X)+1
\end{array}\right)=\left(\begin{array}{c}
N-I D X(P X)+P B(P X)+1 \\
P B(P X)+1
\end{array}\right) \\
\operatorname{SUM}(N, P X)=\operatorname{MIN}(P X)\left(\begin{array}{c}
N \\
I D X(P X)
\end{array}\right)
\end{gathered}
$$

Definition: Subdividethe $\left(K_{1}, K_{2}, \cdots, K_{M}\right)$ by interval of adjacent numbers. If the discontinuity interval is $D>1$, the interval of adjacent numbers $\geq D$ is classified into a same catelog. Use the symbol $P Y$ represent a catelog and represented by [min item]

For example:

$$
\begin{gathered}
P Y=[1,3], \quad(1,3),(1,4),(2,4),(1,5),(2,5),(3,5) \in P Y . \text { Same as } P X=B \\
P Y=[1,4], \quad(1,4),(1,5),(2,5),(1,6),(2,6),(3,6) \in P Y, \quad(3,5),(4,6) \notin P Y \\
P Y=[1,4,6], \quad(1,4,7),(1,5,7),(2,5,7) \in P Y, \quad(3,5,7) \notin P Y
\end{gathered}
$$

Redefinition: $P B(P Y)=$ Count of discontinuity intervals in $P Y$, compatible with $P X$

Redefinition: $I D X(P Y)=$ The maximum factor of $M I N(P Y)+1$, compatible with $P X$

Definition: $B A S E(P Y)=P X$, If $P B(P X)=P B(P Y), P M(P X)=P M(P Y), P X$ has discontinuity intervals at the same positions of $P Y$.

For example:

$$
\begin{gathered}
P Y=[1,3],[1,4],[1, K>2] ; \quad \operatorname{BASE}(P Y)=[1,3]=B \\
P Y=[1,3,4],[1,4,5],[1, K>2, X=K+1] ; \quad \operatorname{BASE}(P Y)=[1,3,4]=B A \\
P Y=[1,3,5],[1,4,9],[1, K>2, X>K+1] ; \operatorname{BASE}(P Y)=[1,3,5]=B B \\
\text { Definition: if } f(n)=\sum K_{i}\left(\begin{array}{c}
N-n_{i} \\
m_{i}
\end{array}\right), \text { then } D^{1} f(n)=\sum K_{i}\left(\begin{array}{c}
N-n_{i}-1 \\
m_{i}-1
\end{array}\right)
\end{gathered}
$$

Definition: $P H(P Y)=I D X(P Y)-P B(P Y)-2=$ Maximum factor of $M I N(P Y)-P B(P Y)-1$ 


$$
\begin{gathered}
P Y=\left[1, K_{1} \cdots K_{M}\right], \quad B S=B A S E(P Y)=\left[1, G_{1} \cdots G_{M}\right] \rightarrow \\
P H(P Y)=K_{M}-I D X(B S)+P M(B S)
\end{gathered}
$$

[Proof]

$$
\begin{aligned}
& P H(P Y)=K_{M}-P B(P Y)-1=K_{M}-P B(B S)-1=K_{M}-G_{M}+M \\
&=K_{M}-\left(G_{M}+1\right)+(M+1)=\text { Right } \\
& \sum_{n=K}^{N-1} n\left(\begin{array}{c}
n-K \\
M
\end{array}\right)=(M+1)\left(\begin{array}{l}
N-K \\
M+2
\end{array}\right)+(M+K)\left(\begin{array}{c}
N-K \\
M+1
\end{array}\right)
\end{aligned}
$$

[Proof]

$$
\begin{aligned}
& \text { Left }=\sum_{n=K}^{N-1}(n-K+1)\left(\begin{array}{c}
n-K \\
M
\end{array}\right)+(K-1) \sum_{n=K}^{N-1}\left(\begin{array}{c}
n-K \\
M
\end{array}\right) \\
& =\sum_{n=K}^{N-1}(M+1)\left(\begin{array}{c}
n-K+1 \\
M+1
\end{array}\right)+(K-1) \sum_{n=K}^{N-1}\left(\begin{array}{c}
n-K \\
M
\end{array}\right) \\
& =(M+1)\left(\begin{array}{c}
N-K+1 \\
M+2
\end{array}\right)+(K-1)\left(\begin{array}{c}
N-K \\
M+1
\end{array}\right) \\
& =(M+1)\left(\begin{array}{c}
N-K \\
M+2
\end{array}\right)+(M+1)\left(\begin{array}{c}
N-K \\
M+1
\end{array}\right)+(K-1)\left(\begin{array}{c}
N-K \\
M+1
\end{array}\right)=\text { Right }
\end{aligned}
$$

By definition:

$$
\operatorname{SUM}(N, P Y)=\sum_{n=0}^{N} \sum E N D(n, P Y)
$$

Derived from (1.2)

$$
\sum \operatorname{END}(N, P Y)=D^{1} S U M(N, P Y)
$$

By definition:

$$
\begin{gathered}
\operatorname{SUM}(N,[1, \cdots, K, K+1])=\sum_{n=0}^{N-1} n \times \sum \operatorname{END}(n,[1, \cdots, K]) \\
\operatorname{SUM}(N,[1, \cdots, K, X>K+1])=\sum_{n=0}^{N-1} n \times \operatorname{SUM}(n-X+K+1,[1, \cdots, K])
\end{gathered}
$$

According to the method in [1]:

$$
\begin{aligned}
\text { Items in } S U M(N, P Y) \mid & =\left(\begin{array}{c}
N-I D X(P Y)+P B(P Y)+1 \\
P B(P Y)+1
\end{array}\right) \\
& =\left(\begin{array}{c}
N-P H(P Y)-1 \\
P B(P Y)+1
\end{array}\right),
\end{aligned}
$$

compatible with (1).

\section{Calculation Formula}

If $P Y=B A S E(P Y)$, the calculation formula has been given by (2).

Otherwise, it can be deduced from (1.1)-(1.5).

$$
\operatorname{SUM}(N,[1, K \geq 3])=3\left(\begin{array}{c}
N-K+2 \\
4
\end{array}\right)+K\left(\begin{array}{c}
N-K+2 \\
3
\end{array}\right)
$$


[Proof]

$$
\begin{aligned}
& \text { Left } \stackrel{1.2)}{\longrightarrow} \sum_{n=0}^{N} \sum \operatorname{END}(n,[1, K])=\sum_{n=K+1}^{N}(n-1)(1+2+\cdots+(n-K)) \\
& =\sum_{n=K+1}^{N}(n-1)\left(\begin{array}{c}
n-K+1 \\
2
\end{array}\right)=\sum_{n=K}^{N-1} n\left(\begin{array}{c}
n-K+2 \\
2
\end{array}\right) \stackrel{1.1)}{\longrightarrow} \text { Right } \\
& \quad \operatorname{SUM}(N,[1,2, K>3])=2 \times 4\left(\begin{array}{c}
N-K+3 \\
5
\end{array}\right)+2 \times K\left(\begin{array}{c}
N-K+3 \\
4
\end{array}\right)
\end{aligned}
$$

[Proof]

$$
\begin{aligned}
& \stackrel{1.5)}{\longrightarrow} \sum_{n=0}^{N-1} n \times \operatorname{SUM}(n-K+3,[1,2]) \\
& \stackrel{(2)}{\longrightarrow} \sum_{n=0}^{N-1} n \times 2\left(\begin{array}{c}
n-K+3 \\
3
\end{array}\right) \stackrel{1.1)}{\longrightarrow} \text { Right } \\
& \operatorname{SUM}(N,[1, K \geq 3, X=K+1]) \\
& =12\left(\begin{array}{c}
N-X+2 \\
5
\end{array}\right)+3(X+1)\left(\begin{array}{c}
N-X+2 \\
4
\end{array}\right) \\
& +3 K\left(\begin{array}{c}
N-X+2 \\
4
\end{array}\right)+K X\left(\begin{array}{c}
N-X+2 \\
3
\end{array}\right)
\end{aligned}
$$

[Proof]

$$
\begin{aligned}
& \stackrel{\text { 1.2) 1.4) 2.1) }}{\longrightarrow} \sum_{n=0}^{N-1} n \times D^{1}\left[3\left(\begin{array}{c}
n-K+2 \\
4
\end{array}\right)+K\left(\begin{array}{c}
n-K+2 \\
3
\end{array}\right)\right] \\
& =\sum_{n=0}^{N-1} n \times\left[3\left(\begin{array}{c}
n-K+1 \\
3
\end{array}\right)+K\left(\begin{array}{c}
n-K+1 \\
2
\end{array}\right)\right] \stackrel{1.1)}{\longrightarrow} \\
& =12\left(\begin{array}{c}
N-K+1 \\
5
\end{array}\right)+3(K+2)\left(\begin{array}{c}
N-K+1 \\
4
\end{array}\right)+3 K\left(\begin{array}{c}
N-K+1 \\
4
\end{array}\right) \\
& +K(K+1)\left(\begin{array}{c}
N-K+1 \\
3
\end{array}\right)
\end{aligned}
$$

$=$ Right

$$
\begin{aligned}
& \operatorname{SUM}(N,[1, K \geq 3, X>K+1]) \\
& =15\left(\begin{array}{c}
N-X+3 \\
6
\end{array}\right)+3(X+1)\left(\begin{array}{c}
N-X+3 \\
5
\end{array}\right) \\
& +4 K\left(\begin{array}{c}
N-X+3 \\
5
\end{array}\right)+K X\left(\begin{array}{c}
N-X+3 \\
4
\end{array}\right)
\end{aligned}
$$

[Proof]

$$
\stackrel{1.52 .1)}{\longrightarrow} \sum_{n=0}^{N-1} n \times\left[3\left(\begin{array}{c}
n-X+3 \\
4
\end{array}\right)+K\left(\begin{array}{c}
n-X+3 \\
3
\end{array}\right)\right] \stackrel{1.1)}{\longrightarrow} \text { Right }
$$

2.1. $\operatorname{SUM}\left(N,\left[1, K_{1} \geq 3, \cdots, K_{M}\right]\right)$

$$
P Y=\left[1, K_{1} \geq 3, \cdots, K_{M}\right], \quad \operatorname{BASE}(P Y)=B S=\left[1, G_{1}, \cdots, G_{M}\right]
$$

$\left.1^{\star}\right) \operatorname{SUM}(N, P Y)=\sum A_{i}\left(\begin{array}{c}P \\ M_{i}\end{array}\right), 2^{M}$ items in total. 
$\left.2^{\star}\right) \quad M_{i}=I D X(B S), I D X(B S)-1, \cdots, I D X(B S)-M$

$\left.3^{*}\right) \quad P=N-P H(P Y)$

Use the form $\left(G_{1}+K_{1}\right)\left(G_{2}+K_{2}\right) \cdots\left(G_{M}+K_{M}\right)=\sum X_{1} X_{2} \cdots X_{M}$. The expansion function has $2^{M}$ items in total.

$\left.4^{*}\right) \quad M_{i}=I D X(B S)-($ Count of $X \in K)$

$\left.5^{\star}\right) A_{i}=\prod_{i=1}^{M}\left(X_{i}+D_{i}\right)$,

$$
D_{i}= \begin{cases}-m, X_{i} \in G, m=\text { count of }\left\{X_{i} \cdots X_{i-1}\right\} \in K & \left.5.1^{*}\right) \\ +m, X_{i} \in K, m=\text { count of }\left\{X_{i} \cdots X_{i-1}\right\} \in G & \left.5.2^{*}\right)\end{cases}
$$

[Proof]

$$
P Y=\left[1, K_{1} \geq 3, \cdots, K_{M}\right], \quad B S=\operatorname{BASE}(P Y)
$$

Suppose $\operatorname{SUM}(N, P Y)=\sum X_{1} \cdots X_{M}\left(\begin{array}{c}P \\ M_{i}\end{array}\right), P=N-P H(P Y)$.

According to inductive hypothesis:

$$
\begin{aligned}
& \left(G_{1}+K_{1}\right)\left(G_{2}+K_{2}\right) \cdots\left(G_{M}+K_{M}\right)\left\{\left(G_{M}+1\right)+\left(K_{M}+1\right)\right\} \\
& =\sum X_{1} X_{2} \cdots X_{M}\left\{\left(G_{M}+1\right)+\left(K_{M}+1\right)\right\} \\
& C=\text { Count of } X \in K, M-C=\text { Count of } X \in G, M_{i}=I D X(B S)-C \\
& P Y 1=\left[P Y, K_{M}+1\right], \quad B S 1=B A S E(P Y 1) \\
& \operatorname{SUM}(N, P Y 1) \stackrel{1,2) 1.3)}{\longrightarrow} \sum_{n=0}^{N-1} n \times D^{1} S U M(n, P Y) \\
& =\sum_{n=0}^{N-1} n \times \sum X_{1} \cdots X_{M}\left(\begin{array}{c}
P-1 \\
M_{i}-1
\end{array}\right) \stackrel{1.1)}{\longrightarrow} \\
& =\sum\left(X_{1} \cdots X_{M} M_{i}\left(\begin{array}{c}
P-1 \\
M_{i}+1
\end{array}\right)+X_{1} \cdots X_{M}\left(P H(P Y)+M_{i}\right)\left(\begin{array}{c}
P-1 \\
M_{i}
\end{array}\right)\right) \\
& =\sum X_{1} \cdots X_{M}(I D X(B S)-C)\left(\begin{array}{c}
P-1 \\
M_{i}+1
\end{array}\right) \\
& +\sum X_{1} \cdots X_{M}(P H(P Y)+I D X(B S)-C)\left(\begin{array}{c}
P-1 \\
M_{i}
\end{array}\right) \\
& =\sum X_{1} \cdots X_{M}\left(\left(G_{M}+1\right)-C\right)\left(\begin{array}{c}
P-1 \\
M_{i}+1
\end{array}\right) \\
& +\sum X_{1} \cdots X_{M}\left(\left(K_{M}+1\right)+(P M(B S)-1)-C\right)\left(\begin{array}{c}
P-1 \\
M_{i}
\end{array}\right) \\
& =\sum X_{1} \cdots X_{M}\left(G_{M+1}-C\right)\left(\begin{array}{c}
P-1 \\
I D X(B S)-C+1
\end{array}\right) \\
& +\sum X_{1} \cdots X_{M}\left(K_{M+1}+M-C\right)\left(\begin{array}{c}
P-1 \\
I D X(B S)-C
\end{array}\right) \\
& =\sum X_{1} \cdots X_{M}\left(G_{M+1}-C\right)\left(\begin{array}{c}
P-1 \\
I D X(B S 1)-C
\end{array}\right) \\
& +\sum X_{1} \cdots X_{M}\left(K_{M+1}+M-C\right)\left(\begin{array}{c}
P-1 \\
I D X(B S 1)-(C+1)
\end{array}\right)
\end{aligned}
$$


$\left.1^{*}\right)$ is obvious.

$M_{i}$ change form IDX $(B S), \cdots, I D X(B S)-M$ to

$I D X(B S)+1, \cdots, I D X(B S)-M=I D X(B S 1), \cdots, I D X(B S 1)-(M+1)$

$\left.2^{\star}\right)$ proved

$$
\begin{aligned}
P-1 & =N-P H(P Y)-1=N-\left(K_{M}-I D X(B S)+P M(B S)\right)-1 \\
& =N-\left\{\left(K_{M}+1\right)-(I D X(B S)+1)+(P M(B S)+1)\right\} \\
& =N-\left(K_{M+1}-I D X(B S 1)+P M(B S 1)\right)=N-P H(P Y 1)
\end{aligned}
$$

$3^{\star}$ ) proved

$$
\begin{gathered}
\left.\left.X_{1} \cdots X_{M}\left(G_{M+1}-C\right)\left(\begin{array}{c}
P-1 \\
I D X(B S 1)-C
\end{array}\right) \rightarrow 4^{\star}\right) 5.1^{\star}\right) \\
\left.\left.X_{1} \cdots X_{M}\left(G_{M+1}-C\right)\left(\begin{array}{c}
P-1 \\
I D X(B S 1)-(C+1)
\end{array}\right) \rightarrow 4^{\star}\right) 5.2^{\star}\right)
\end{gathered}
$$

$\left.4^{\star}\right) 5^{\star}$ ) proved

$$
\begin{aligned}
& P Y 1=\left[P Y, K_{M+1}>K_{M}+1\right], \quad B S 1=\operatorname{BASE}(P Y 1) \\
& \operatorname{SUM}(N, P Y 1) \stackrel{1.5)}{\longrightarrow} \sum_{n=0}^{N-1} n \times \operatorname{SUM}\left(n-K_{M+1}+K_{M}+1, P Y\right) \\
& =\sum_{n=0}^{N-1} n \times \sum X_{1} \cdots X_{M}\left(\begin{array}{c}
n-K_{M+1}+K_{M}+1-P H(P Y) \\
M_{i}
\end{array}\right) \\
& =\sum_{n=0}^{N-1} n \times \sum X_{1} \cdots X_{M}\left(\begin{array}{c}
n-K_{M+1}+K_{M}+1-\left(K_{M}-I D X(B S)+P M(B S)\right) \\
M_{i}
\end{array}\right) \\
& =\sum_{n=0}^{N-1} n \times \sum X_{1} \cdots X_{M}\left(\begin{array}{c}
n-\left(K_{M+1}-I D X(B S)+P M(B S)-1\right) \\
M_{i}
\end{array}\right) \\
& =\sum X_{1} \cdots X_{M}\left(M_{i}+1\right)\left(\begin{array}{c}
P 1 \\
M_{i}+2
\end{array}\right) \\
& +\sum X_{1} \cdots X_{M}\left(K_{M+1}-I D X(B S)+M+M_{i}\right)\left(\begin{array}{c}
P 1 \\
M_{i}+1
\end{array}\right) \\
& =\sum X_{1} \cdots X_{M}(I D X(B S)-C+1)\left(\begin{array}{c}
P 1 \\
M_{i}+2
\end{array}\right) \\
& +\sum X_{1} \cdots X_{M}\left(K_{M+1}+M-C\right)\left(\begin{array}{c}
P 1 \\
M_{i}+1
\end{array}\right) \\
& =\sum X_{1} \cdots X_{M}\left(G_{M+1}-C\right)\left(\begin{array}{c}
P 1 \\
I D X(B S)-C+2
\end{array}\right) \\
& +\sum X_{1} \cdots X_{M}\left(K_{M+1}+M-C\right)\left(\begin{array}{c}
P 1 \\
I D X(B S)-C+1
\end{array}\right) \\
& =\sum X_{1} \cdots X_{M}\left(G_{M+1}-C\right)\left(\begin{array}{c}
P 1 \\
I D X(B S 1)-C
\end{array}\right) \\
& +\sum X_{1} \cdots X_{M}\left(K_{M+1}+M-C\right)\left(\begin{array}{c}
P 1 \\
I D X(B S 1)-(C+1)
\end{array}\right)
\end{aligned}
$$

$\left.1^{\star}\right)$ is obvious. 
$M_{i}$ change form $\operatorname{IDX}(B S), \cdots, I D X(B S)-M$ to

$I D X(B S)+2, \cdots, I D X(B S)-M+1=I D X(B S 1), \cdots, I D X(B S 1)-(M+1)$

$\left.2^{\star}\right)$ proved

$$
P 1=N-\left\{K_{M+1}-(I D X(B S)+2)+(P M(B S)+1)\right\}=N-P H(P Y 1)
$$

$3^{*}$ ) proved

$$
\begin{gathered}
\left.\left.X_{1} \cdots X_{M}\left(G_{M+1}-C\right)\left(\begin{array}{c}
P 1 \\
I D X(B S 1)-C
\end{array}\right) \rightarrow 4^{\star}\right) 5.1^{\star}\right) \\
\left.\left.X_{1} \cdots X_{M}\left(K_{M+1}+M-C\right)\left(\begin{array}{c}
P 1 \\
I D X(B S 1)-(C+1)
\end{array}\right) \rightarrow 4^{\star}\right) 5.2^{\star}\right)
\end{gathered}
$$

$\left.4^{\star}\right) 5^{\star}$ proved

q.e.d.

Example 2.1:

$$
\begin{array}{rl} 
& \left(3+K_{1}\right)\left(5+K_{2}\right)\left(7+K_{3}\right) \\
= & 3 \times 5 \times 7+3 \times 5 \times K_{3}+3 \times K_{2} \times 7+3 \times K_{2} \times K_{3}+K_{1} \times 5 \times 7 \\
& +K_{1} \times 5 \times K_{3}+K_{1} \times K_{2} \times 7+K_{1} \times K_{2} \times K_{3} \\
P=N- & K_{3}+I D X([1,3,5,7])-P M([1,3,5,7])=N-K_{3}+8-4=N-K_{3}+4 \\
S & U M\left(N,\left[1, K_{1} \geq 3, K_{2} \geq K_{1}+2, K_{3} \geq K_{2}+2\right]\right) \\
= & 3 \times 5 \times 7\left(\begin{array}{l}
P \\
8
\end{array}\right)+3 \times 5 \times\left(K_{3}+2\right)\left(\begin{array}{l}
P \\
7
\end{array}\right)+3 \times\left(K_{2}+1\right) \times(7-1)\left(\begin{array}{l}
P \\
7
\end{array}\right) \\
+ & 3 \times\left(K_{2}+1\right) \times\left(K_{3}+1\right)\left(\begin{array}{l}
P \\
7
\end{array}\right)+K_{1} \times(5-1) \times(7-1)\left(\begin{array}{l}
P \\
7
\end{array}\right) \\
+ & K_{1} \times(5-1) \times\left(K_{3}+1\right)\left(\begin{array}{l}
P \\
6
\end{array}\right)+K_{1} \times K_{2} \times(7-2)\left(\begin{array}{l}
P \\
6
\end{array}\right) \\
+ & K_{1} \times K_{2} \times K_{3}\left(\begin{array}{l}
P \\
5
\end{array}\right)
\end{array}
$$

2.2. $\operatorname{SUM}\left(N,\left[1,2, \cdots, n, K_{1} \geq n+2, \cdots, K_{M}\right]\right)$

$$
\begin{gathered}
P Y=\left[1,2,3, \cdots, n, K_{1} \geq n+2, \cdots, K_{M}\right], \\
\operatorname{BASE}(P Y)=B S=\left[1,2,3, \cdots, n, G_{1}, \cdots, G_{M}\right]
\end{gathered}
$$

$\left.1^{\star}\right) \operatorname{SUM}(N, P Y)=\sum A_{i}\left(\begin{array}{c}P \\ M_{i}\end{array}\right), 2^{M}$ items in total.

2*) $M_{i}=I D X(B S), I D X(B S)-1, \cdots, I D X(B S)-M$

$\left.3^{\star}\right) \quad P=N-K_{M}+I D X(B S)-(M+1)=N-\left(K_{M}-P B(P Y)-1\right)=N-P H(P Y)$

Use the form $\left(G_{1}+K_{1}\right)\left(G_{2}+K_{2}\right) \cdots\left(G_{M}+K_{M}\right)=\sum X_{1} X_{2} \cdots X_{M}$.

$\left.4^{\star}\right) \quad M_{i}=I D X(B S)-($ Count of $X \in K)$

$\left.5^{\star}\right) \quad A_{i}=n ! \times \quad($ Same as 2.1)

Example 2.2:

$$
\left(5+K_{1}\right)\left(7+K_{2}\right)=5 \times 7+5 \times K_{2}+K_{1} \times 7+K_{1} \times K_{2},
$$




$$
\begin{gathered}
P=N-K_{2}+\operatorname{IDX}([1,2,3,5,7])-3=N-K_{2}+5 \\
\operatorname{SUM}\left(N,\left[1,2,3, K_{1} \geq 5, K_{2} \geq K_{1}+2\right]\right) \\
=3 !\left\{5 \times 7\left(\begin{array}{l}
P \\
8
\end{array}\right)+5 \times\left(K_{2}+1\right)\left(\begin{array}{l}
P \\
7
\end{array}\right)+K_{1} \times(7-1)\left(\begin{array}{l}
P \\
7
\end{array}\right)+K_{1} K_{2}\left(\begin{array}{l}
P \\
6
\end{array}\right)\right\}
\end{gathered}
$$

\subsection{The Meaning of the Expansion of SUM (N, PY)}

$$
P Y=\left[1, K_{1}, \cdots, K_{M}\right], \text { an Item }=\left\{\text { begin, } K_{1}+E_{1}, \cdots, K_{M}+E_{M}\right\}
$$

$($ product of an item $)=\operatorname{begin} \times\left(K_{1}+E_{1}\right) \cdots\left(K_{M}+E_{M}\right)=\operatorname{begin} \times \sum F_{1} \cdots F_{M}$

$F_{i}=E \quad\left(\right.$ means $\left.F_{i}=E_{i}\right)$ or $F_{i}=K_{i}$,

$$
\operatorname{SUM}(N, P Y)=\sum \text { product }=\sum \sum \text { begin } \times F_{1} \cdots F_{M}
$$

Define 2.3. $S U M \_K\left(N, P Y, P F=F_{1} F_{2} \cdots F_{M}\right)=\sum$ Items in $\left(2.3 .1^{\star}\right)$ with the same $P F$

Example 2.3:

$$
\begin{aligned}
& \operatorname{SUM}\left(N,\left[1, K_{1} \geq 3, K_{2} \geq K_{1}+2\right]\right) \\
= & 15\left(\begin{array}{c}
N-K_{2}+3 \\
6
\end{array}\right)+3\left(K_{2}+1\right)\left(\begin{array}{c}
N-K_{2}+3 \\
5
\end{array}\right) \\
& +4 K_{1}\left(\begin{array}{c}
N-K_{2}+3 \\
5
\end{array}\right)+K_{1} K_{2}\left(\begin{array}{c}
N-K_{2}+3 \\
4
\end{array}\right) \\
= & 15\left(\begin{array}{c}
N-K_{2}+3 \\
6
\end{array}\right)+3\left(\begin{array}{c}
N-K_{2}+3 \\
5
\end{array}\right)+3 K_{2}\left(\begin{array}{c}
N-K_{2}+3 \\
5
\end{array}\right) \\
& +4 K_{1}\left(\begin{array}{c}
N-K_{2}+3 \\
5
\end{array}\right)+K_{1} K_{2}\left(\begin{array}{c}
N-K_{2}+3 \\
4
\end{array}\right) \\
= & \sum_{n=0}^{N-K_{2}} \sum n \times\left(K_{1}+E_{1, i}\right)\left(K_{2}+E_{2, i}\right)
\end{aligned}
$$

then can prove

$$
\begin{gathered}
S U M \_K(N, P Y, E E) \\
=\sum_{\text {all items }} \text { begin } * E_{1, i} E_{2, i}=15\left(\begin{array}{c}
N-K_{2}+3 \\
6
\end{array}\right)+3\left(\begin{array}{c}
N-K_{2}+3 \\
5
\end{array}\right) \\
S U M \_K\left(N, P Y, K_{1} E\right)=\sum_{\text {all items }} \text { begin } * K_{1} E_{2, i}=4 K_{1}\left(\begin{array}{c}
N-K_{2}+3 \\
5
\end{array}\right) \\
S U M \_K\left(N, P Y, E K_{2}\right)=\sum_{\text {all items }} \text { begin } * E_{1, i} K_{2}=3 K_{2}\left(\begin{array}{c}
N-K_{2}+3 \\
5
\end{array}\right) \\
S U M \_K\left(N, P Y, K_{1} K_{2}\right)=\sum_{\text {all items }} \text { begin } * K_{1} K_{2}=K_{1} K_{2}\left(\begin{array}{c}
N-K_{2}+3 \\
4
\end{array}\right) \\
S U M(N, P Y)=\sum \prod_{i=1}^{M}\left(X_{i}+D_{i}\right)\left(\begin{array}{c}
P \\
M_{i}
\end{array}\right),
\end{gathered}
$$




$$
X_{i}+D_{i}=\left\{\begin{array}{l}
\left\{G_{i}-D_{i}\right\}, X_{i} \in G, D_{i}=\text { count of }\left\{X_{1} \cdots X_{i-1}\right\} \in K \\
\left\{K_{i}\right\}+\left\{D_{i}\right\}, X_{i} \in K, D_{i}=\text { count of }\left\{X_{1} \cdots X_{i-1}\right\} \in G
\end{array}\right.
$$

Theorem 2.3 SUM_K $(N, P Y, P F)=\sum$ Items in $\left(2.3 .2^{*}\right)$ expand by \{\} , factors has the same $\left\{K_{i}\right\} \in F$

$$
\begin{gathered}
=\sum \prod_{i=1}^{M} Y_{i}\left(\begin{array}{c}
P \\
M_{i}
\end{array}\right), \\
Y_{i}=\left\{\begin{array}{l}
0, F_{i} \in K, X_{i} \in G \\
K_{i}, F_{i} \in K, X_{i} \in K \\
G_{i}-D_{i}, F_{i}=E, X_{i} \in G, D_{i}=\text { count of }\left\{\cdots X_{i-1}\right\} \in K \\
D_{i}, F_{i}=E, X_{i} \in K, D_{i}=\text { count of }\left\{\cdots X_{i-1}\right\} \in G
\end{array}\right. \\
P=N-P H(P Y), M_{i}=I D X(B S)-(\text { Count of } X \in K)
\end{gathered}
$$

[Proof]

Suppose $S U M_{-} K(N, P Y, P Z)=\sum \prod_{i=1}^{M} Y_{i}\left(\begin{array}{c}P \\ M_{i}\end{array}\right), \quad P=N-P H(P Y)$.

$$
P Y 1=\left[P Y, K_{M+1}\right], \quad C=\text { Count of } X \in K,
$$$$
M-C=\text { Count of } X \in G, M_{i}=I D X(B S)-C
$$

When $K_{M+1}=K_{M}+1$

$$
\begin{aligned}
& S U M(N, P Y 1) \stackrel{1,2) 1.3)}{\longrightarrow} \sum_{n=0}^{N-1} n \times D^{1} S U M(n, P Y) \\
& \rightarrow \sum_{n=0}^{N-1} n \times D^{1} S U M \_K(n, P Y, P F) \\
&= \sum_{n=0}^{N-1} n \times \sum \prod_{i=1}^{M} Y_{i}\left(\begin{array}{c}
P-1 \\
M_{i}-1
\end{array}\right) \stackrel{\text { reference (2.1.1) }}{\longrightarrow} \\
&= \sum \prod_{i=1}^{M} Y_{i}\left(G_{M+1}-C\right)\left(\begin{array}{c}
P-1 \\
I D X(B S 1)-C
\end{array}\right) \\
&+\sum \prod_{i=1}^{M} Y_{i}(M-C)\left(\begin{array}{c}
P-1 \\
I D X(B S 1)-(C+1)
\end{array}\right) \\
&+\sum \prod_{i=1}^{M} Y_{i} K_{M+1}\left(\begin{array}{c}
P-1 \\
I D X(B S 1)-(C+1)
\end{array}\right) \\
&= S U M \_K(N, P Y 1,[P F, E])+S U M \_K\left(N, P Y 1,\left[P F, K_{M+1}\right]\right) \\
& S U M \_K\left(N, P Y 1,\left[P F, K_{M+1}\right]\right)=\sum \prod_{i=1}^{M} Y_{i} K_{M+1}\left(\begin{array}{c}
P D X(B S 1)-(C+1) \\
I D-1=N-P H(P Y 1) \rightarrow
\end{array}\right) \rightarrow \\
& \quad S U M_{-} K(N, P Y 1,[P F, E]) \\
&=\sum \prod_{i=1}^{M} Y_{i}\left(G_{M+1}-C\right)\left(\begin{array}{c}
P-1 \\
I D X(B S 1)-C
\end{array}\right) \\
&+\sum \prod_{i=1}^{M} Y_{i}(M-C)\left(\begin{array}{c}
P-1 \\
I D X(B S 1)-(C+1)
\end{array}\right) \rightarrow
\end{aligned}
$$




$$
\begin{aligned}
& \text { When } K_{M+1}>K_{M}+1 \\
& S U M(N, P Y 1) \stackrel{1,5)}{\longrightarrow} \sum_{n=0}^{N-1} n \times S U M\left(n-K_{M+1}+K_{M}+1, P Y\right) \\
& \rightarrow \sum_{n=0}^{N-1} n \times S U M \_K\left(n-K_{M+1}+K_{M}+1, P Y, P F\right) \\
&= \sum_{n=0}^{N-1} n \times \sum \prod_{i=1}^{M} Y_{i}\left(\begin{array}{c}
P 1 \\
M_{i}
\end{array}\right) \stackrel{\text { reference }(2.1 .2)}{\longrightarrow} \\
&= \sum \prod_{i=1}^{M} Y_{i}\left(G_{M+1}-C\right)\left(\begin{array}{c}
P 1 \\
I D X(B S 1)-C
\end{array}\right) \\
&+\sum \prod_{i=1}^{M} Y_{i}(M-C)\left(\begin{array}{c}
P 1 \\
I D X(B S 1)-(C+1)
\end{array}\right) \\
&+\sum \prod_{i=1}^{M} Y_{i} K_{M+1}\left(\begin{array}{c}
P 1 \\
I D X(B S 1)-(C+1)
\end{array}\right) \\
&= S U M \_K(N, P Y 1,[P F, E])+S U M \_K\left(N, P Y 1,\left[P F, K_{M+1}\right]\right) \\
& P U 1=N-P H(P Y 1) \rightarrow\left(2.3 .3^{*}\right) \\
& \text { SUM_K }\left(N, P Y 1,\left[P F, K_{M+1}\right]\right)=\sum \prod_{i=1}^{M} Y_{i} K_{M+1}\left(\begin{array}{c}
I D X(B S 1)-(C+1)
\end{array}\right) \rightarrow\left(2.3 .3^{*}\right) \\
& \quad S U M \_K(N, P Y 1,[P F, E]) \\
&=\sum \prod_{i=1}^{M} Y_{i}\left(G_{M+1}-C\right)\left(\begin{array}{c}
P 1 \\
I D X(B S 1)-C
\end{array}\right) \\
&+\sum \prod_{i=1}^{M} Y_{i}(M-C)\left(\begin{array}{c}
P 1 \\
I D X(B S 1)-(C+1)
\end{array}\right) \rightarrow
\end{aligned}
$$

q.e.d.

2.3.1) SUM_K $\left(N, P Y=\left[1,2, \cdots, n, K_{1} \geq n+2, \cdots, K_{M}\right], P F\right)$ has the similar conclusions.

2.3.2) SUM_K $\left(N, P Y=\left[1,2, \cdots, n, K_{1} \geq n+2, \cdots, K_{M}\right], K_{1} K_{2} \cdots K_{M}\right)$,

$$
\begin{aligned}
B S & =B A S E(P Y)=M I N(P Y)\left(\begin{array}{c}
N-P H(P Y) \\
I D X(B S)-M
\end{array}\right) \\
& =M I N(P Y) \sum_{n=0}^{N-1}\left(\begin{array}{c}
n-P H(P Y)-1 \\
I D X(B S)-M-1
\end{array}\right) \\
& =\operatorname{MIN}(P Y) \sum_{n=0}^{N-1}\left(\begin{array}{c}
n-P H(P Y)-1 \\
P B(B S)+1
\end{array}\right) \\
& =\operatorname{MIN}(P Y) \sum_{n=0}^{N-1}\left(\begin{array}{c}
n-P H(P Y)-1 \\
P B(P Y)+1
\end{array}\right) \\
& =\operatorname{MIN}(P Y) \sum_{n=0}^{N-1}|\operatorname{Items~in~} S U M(n+1, P Y)|
\end{aligned}
$$

\section{A Theorem of Ring}

$$
\begin{gathered}
P Y=\left[1, K_{1} \geq 3, \cdots, K_{M}\right], \quad \operatorname{BASE}(P Y)=B S=\left[1, G_{1}, \cdots, G_{M}\right] \\
\operatorname{SUM}(N, P Y)=\sum A_{i}\left(\begin{array}{c}
P \\
M_{i}
\end{array}\right),
\end{gathered}
$$


When $P Y=B S, \quad S U M(N, P Y)=\operatorname{MIN}(B S)\left(\begin{array}{c}N \\ I D X(B S)\end{array}\right)$

This inspired us:

In the form $\left(G_{1}+K_{1}\right)\left(G_{2}+K_{2}\right) \cdots\left(G_{M}+K_{M}\right), \quad K_{i}=G_{i} \rightarrow \operatorname{MIN}(B S) \mid \sum A_{i}$ ( $M_{i}$ is same).

Definition 3.1:

$S,\left\{K_{i}\right\} \in \operatorname{Ring} ; K_{i}=K_{j}, K_{i}<K_{j}, K_{i}>K_{j}$ are allowed.

Choice $N$ from $\left(K_{1}, K_{2}, \cdots, K_{M}\right), R=R_{1} \cdots R_{M}$

$R_{i}=+1$ Indicates that $K_{i}$ was selected, $R_{i}=-1$ Indicates that $K_{i}$ was unselected,

$$
\begin{gathered}
F(N, M, R, K, S)=\prod_{i=1}^{M}\left(K_{i}+D_{i}\right), \\
D_{i}=\left\{\begin{array}{l}
-m S, R_{i}=-1, m=\text { count of }\left\{K_{1}, \cdots, K_{i-1}\right\} \text { selected } \\
+m S, R_{i}=+1, m=\text { count of }\left\{K_{1}, \cdots, K_{i-1}\right\} \text { unselected }
\end{array}\right.
\end{gathered}
$$

When $S=1$, abbreviated as $F(N, M, R, K)$

\section{Definition 3.2:}

$H\left(N_{1}, N_{2}, K, S\right)=\sum F\left(N_{1}, N_{1}+N_{2}, R, K, S\right)$, Sum traverses all $N_{1}$-Choice of $K$

Theorem 3.1: $H\left(N_{1}, N_{2}, K, S\right)=\left(\begin{array}{c}M \\ N_{1}, N_{2}\end{array}\right) K_{1} \cdots K_{M}, \quad M=N_{1}+N_{2}$

[Proof]

When $N_{1}=0$ or $N_{2}=0$, it is obvious.

$$
\begin{aligned}
& H(1,1, K, S)=\text { select } K_{1}+\text { select } K_{2} \\
& \text { = unselect } K_{2}+\text { select } K_{2} \\
& =K_{1}\left(K_{2}-S\right)+K_{1}\left(K_{2}+S\right) \\
& =2 K_{1} K_{2} \\
& \text { Suppose } H(1, M-1, K, S)=\left(\begin{array}{c}
M \\
1
\end{array}\right) K_{1} \cdots K_{M} \\
& H(1, M, K, S) \\
& \text { = select } K_{M+1}+\text { unselect } K_{M+1} \\
& =H(0, M, K, S)\left(K_{M+1}+M S\right)+H(1, M-1, K, S)\left(K_{M+1}-S\right) \\
& =\left(\begin{array}{c}
M \\
0
\end{array}\right) K_{1} \cdots K_{M}\left(K_{M+1}+M S\right)+\left(\begin{array}{c}
M \\
1
\end{array}\right) K_{1} \cdots K_{M}\left(K_{M+1}-S\right) \\
& =\left\{\left(\begin{array}{c}
M \\
0
\end{array}\right)+\left(\begin{array}{c}
M \\
1
\end{array}\right)\right\} K_{1} \cdots K_{M} K_{M+1}+\left\{M\left(\begin{array}{c}
M \\
0
\end{array}\right)-\left(\begin{array}{c}
M \\
1
\end{array}\right)\right\} S \times K_{1} \cdots K_{M} K_{M+1} \\
& =\left(\begin{array}{c}
M+1 \\
1
\end{array}\right) K_{1} \cdots K_{M} K_{M+1} \\
& \rightarrow H\left(1, N_{2}, K, S\right) \text { holds } \rightarrow \text { Symmetry } \rightarrow H\left(N_{2}, 1, K, S\right) \text { holds } \\
& \text { Suppose } H\left(N_{1}, M-N_{1}, K, S\right)=\left(\begin{array}{c}
M \\
N_{1}
\end{array}\right) K_{1} \cdots K_{M}
\end{aligned}
$$




$$
\begin{aligned}
& H\left(N_{1}+1, M-N_{1}, K, S\right)=\text { select } K_{M+1}+\text { unselect } K_{M+1} \\
& =H\left(N_{1}, M-N_{1}, K, S\right)\left(K_{M+1}+\left(M-N_{1}\right) S\right) \\
& +H\left(N_{1}+1, M-N_{1}-1, K, S\right)\left(K_{M+1}-\left(N_{1}+1\right) S\right) \\
& =\left(\begin{array}{l}
M \\
N_{1}
\end{array}\right) K_{1} \cdots K_{M}\left(K_{M+1}+\left(M-N_{1}\right) S\right) \\
& +\left(\begin{array}{c}
M \\
N_{1}+1
\end{array}\right) K_{1} \cdots K_{M}\left(K_{M+1}-\left(N_{1}+1\right) S\right) \\
& =\left\{\left(\begin{array}{c}
M \\
N_{1}
\end{array}\right)+\left(\begin{array}{c}
M \\
N_{1}+1
\end{array}\right)\right\} K_{1} \cdots K_{M+1} \\
& +\left\{\left(M-N_{1}\right)\left(\begin{array}{c}
M \\
N_{1}
\end{array}\right)-\left(N_{1}+1\right)\left(\begin{array}{c}
M \\
N_{1}+1
\end{array}\right)\right\} S \times K_{1} \cdots K_{M+1} \\
& =\left(\begin{array}{c}
M+1 \\
N_{1}+1
\end{array}\right) K_{1} \cdots K_{M} K_{M+1} \\
& \rightarrow H\left(N_{1}, N_{2}, K, S\right) \text { holds } \\
& H(1,2, K)=\text { select } A+\text { select } B+\text { select } C \\
& =A(B-1)(C-1)+A(B+1)(C-1)+A B(C+2) \\
& =3 A B C \\
& H(2,1, K)=\text { select } A B+\text { select } B C+\text { select } A C \\
& =A B(C-2)+A(B+1)(C+1)+A(B-1)(C+1) \\
& =3 A B C
\end{aligned}
$$

\section{Definition 3.3:}

$K_{i}$ come from $q$ sources: $S_{1}, S_{2}, \cdots, S_{q}, K_{i} \in S_{j}$ indicates $K_{i}$ come from $S_{j}$ $\operatorname{diff}\left(K_{i}, K_{j}\right)=\operatorname{diff}\left(K_{i} \in S_{a}, K_{j} \in S_{b}\right)=\operatorname{diff}(a, b), \quad \operatorname{diff}(a, b)=S, \quad(a \neq b)$; $\operatorname{diff}(a, b)=-\operatorname{diff}(b, a)$; diff $(a, a)=0$ then can change related parts of definition 3.1 to

$$
\prod_{i=1}^{M}\left(K_{i}+D_{i}\right), D_{i}=\sum_{j<i} \operatorname{diff}\left(K_{i}, K_{j}\right)
$$

and define $H\left(N_{1}, N_{2}, \cdots, N_{q}, K, S\right)$

Theorem 3.2 $H\left(N_{1}, N_{2}, \cdots, N_{q}, K, S\right)=\left(\begin{array}{c}M \\ N_{1}, N_{2}, \cdots, N_{q}\end{array}\right) K_{1} \cdots K_{M}$, $M=N_{1}+N_{2}+\cdots+N_{q}$

[Proof]

Only need to prove $S=1$, and specify $\operatorname{diff}(a, b)=1,(a<b)$ $H\left(N_{1}, N_{2}, K\right)=\sum$ Item,$K_{i} \in\left\{S_{1}, S_{2}\right\}$. An item has M factors,

Choice $N_{1}$ factors, $K_{i}$ in these factors, $K_{i} \in S_{1}$, It is called invariant factor $\{F\}$. Others are called variable factors $\{V\}$ $L_{2}+L_{3}=N_{2}$, choice $L_{2}$ factors in $\{V\}$ to $S_{2}, L_{3}$ to $S_{3}$ By definition, $H\left(L_{2}, L_{3}, V\right)=\sum\left\{\left(L_{2}, L_{3}\right)-\right.$ Choice of $\left.V\right\}$ 


$$
\begin{aligned}
& \sum\left(\prod\{F\} \times \sum\left\{\left(L_{2}, L_{3}\right)-\text { Choice of } V\right\}\right) \\
& =\sum\left(\prod\{F\} \times H\left(L_{2}, L_{3}, V\right)\right)=\sum\left(\prod\{F\} \times\left(\begin{array}{c}
N_{2} \\
L_{1}, L_{2}
\end{array}\right) \times \prod\{V\}\right) \\
& =\left(\begin{array}{c}
N_{2} \\
L_{2}, L_{3}
\end{array}\right) \sum \text { Item }=\left(\begin{array}{c}
N_{2} \\
L_{2}, L_{3}
\end{array}\right)\left(\begin{array}{c}
M \\
N_{1}, N_{2}
\end{array}\right) K_{1} \cdots K_{M} \\
& =\left(\begin{array}{c}
M \\
N_{1}, L_{2}, L_{3}
\end{array}\right) K_{1} \cdots K_{M}=H\left(N_{1}, L_{2}, L_{3}, K\right)
\end{aligned}
$$

and items gives all $\left(N_{1}, L_{2}, L_{3}\right)$-Choice of $K$.

Prove $\sum\left(\prod\{F\} \times \sum\left\{\left(L_{2}, L_{3}\right)\right.\right.$-Choice of $\left.\left.V\right\}\right)$ satisfies the definition of $H\left(N_{1}, \cdots, K\right)$

Let $\quad$ Item $=\prod_{i=1}^{M}\left(K_{i}+D_{i}\right), \quad($ an item changed $)=\prod_{i=1}^{M}\left(H_{i}+E_{i}\right), \quad K_{i}=H_{i}$

$A 1=$ Count of $\left\{K_{1}, \cdots, K_{i-1}\right\} \in S_{1}, \quad A 2=$ Count of $\left\{K_{1}, \cdots, K_{i-1}\right\} \in S_{2}$

$$
\begin{gathered}
B 1=\text { Count of }\left\{\cdots, H_{i-1}\right\} \in S_{1}, \quad B 2=\text { Count of }\left\{\cdots, H_{i-1}\right\} \in S_{2}, \\
B 3=\text { Count of }\left\{\cdots, H_{i-1}\right\} \in S_{3} \\
\rightarrow A 1=B 1, \quad A 2=B 2+B 3 \\
K_{i+1} \in S_{1} \rightarrow H_{i+1} \in S_{1} \rightarrow D_{i+1}=A 2=i-A 1=i-B 1=B 2+B 3=E_{i+1}
\end{gathered}
$$

$\rightarrow$ The invariant factors match the definition

$K_{i+1} \in S_{2}, H_{i+1} \in S_{2} \stackrel{\text { definition of } H\left(L_{2}, L_{3}, V\right)}{\longrightarrow} E_{i}=D_{i}+B 3=-A 1+B 3=-B 1+B 3$ definition of $H\left(N, L_{2}, L_{3}, V\right) \rightarrow$ Match the definition

$K_{i+1} \in S_{2}, H_{i+1} \in S_{3} \stackrel{\text { definition of } H\left(L_{2}, L_{3}, V\right)}{\longrightarrow} E_{i}=D_{i}-B 2=-A 1-B 2=-B 1-B 2$ definition of $H\left(N, L_{2}, L_{3}, V\right) \rightarrow$ Match the definition

$\rightarrow$ Match the definition of $H\left(N_{1}, N_{2}, N_{3}, K\right) \stackrel{\text { recursion }}{\longrightarrow} \cdots$

q.e.d.

Example $3.2\left\{K_{1}, K_{2}, K_{3}\right\}=\{A, B, C\}$

$$
\begin{gathered}
H(1,2, K)=A(B-1)(C-1)+A(B+1)(C-1)+A B(C+2)=3 A B C \\
A(B-1)(C-1) \in S_{1} S_{2} S_{2} \rightarrow\{F\}=\{A\} \rightarrow A\{(B-1)(C-2)+(B-1) C\} \\
\rightarrow A(B-1)(C-2)+A(B-1) C \in S_{1} S_{2} S_{3}+S_{1} S_{3} S_{2} \\
A(B+1)(C-1) \in S_{2} S_{1} S_{2} \rightarrow\{F\}=\{B+1\} \rightarrow(B+1)\{A(C-2)+A C\} \\
\rightarrow A(B+1)(C-2)+A(B+1) C \in S_{2} S_{1} S_{3}+S_{3} S_{1} S_{2} \\
A B(C+2) \in S_{2} S_{2} S_{1} \rightarrow\{F\}=\{C+2\} \rightarrow(C+2)\{A(B-1)+A(B+1)\} \\
\rightarrow A(B-1)(C+2)+A(B+1)(C+2) \in S_{2} S_{3} S_{1}+S_{3} S_{2} S_{1} \\
H(2,1, K)=A B(C-2)+A(B+1)(C+1)+A(B-1)(C+1)=3 A B C \\
A B(C-2) \in S_{1} S_{1} S_{2} \rightarrow\{F\}=\{C-2\} \rightarrow(C-2)\{A(B-1)+A(B+1)\} \\
\rightarrow A(B-1)(C-2)+A(B+1)(C-2) \in S_{1} S_{2} S_{3}+S_{2} S_{1} S_{3}
\end{gathered}
$$




$$
\begin{aligned}
& A(B+1)(C+1) \in S_{2} S_{1} S_{1} \rightarrow\{F\}=\{A\} \rightarrow A\{(B+1) C+(B+1)(C+2)\} \\
& \rightarrow A(B+1) C+A(B+1)(C+2) \in S_{3} S_{1} S_{2}+S_{3} S_{2} S_{1} \\
& A(B-1)(C+1) \in S_{1} S_{2} S_{1} \rightarrow\{F\}=\{B-1\} \rightarrow(B-1)\{A C+A(C+2)\} \\
& \rightarrow A(B-1) C+A(B-1)(C+2) \in S_{1} S_{3} S_{2}+S_{2} S_{3} S_{1} \\
& H(1,1,1, K)=A(B-1)(C-2)+A(B-1) C+A(B+1)(C-2) \\
& +A(B+1) C+A(B-1)(C+2)+A(B+1)(C+2) \\
& =6 A B C
\end{aligned}
$$

3.1) $\left(K_{1}, K_{2}, \cdots, K_{M}\right)$ is permutable in $H\left(N_{1}, N_{2}, \cdots, N_{q}, K, S\right)$

3.2) $H\left(N_{1}, N_{2}, \cdots, N_{q}, K, S\right)=\prod_{i=1}^{M}\left(K_{i}+D_{i}\right)$

$$
\rightarrow H\left(N_{q}, N_{q-1}, \cdots, N_{1}, K, S\right)=\prod_{i=1}^{M}\left(K_{i}-D_{i}\right)
$$

3.3) In the section $2, \operatorname{SUM}(N, P Y)=\sum A_{i}\left(\begin{array}{c}P \\ M_{i}\end{array}\right)$

$A_{i}$ is generated by the form $\left(G_{1}+K_{1}\right)\left(G_{2}+K_{2}\right) \cdots\left(G_{M}+K_{M}\right)$. It can be understood asgenerated by 2-SET: $\left\{G_{i}\right\},\left\{K_{i}\right\}$.

If $K_{i}=J_{1, i}+J_{2, i}+\cdots+J_{x, i},\left(J_{x, i}>0, J_{x, i}=0, J_{x, i}<0\right.$ are allowed),

$$
\operatorname{SUM}(N, P Y)=\sum B_{i}\left(\begin{array}{c}
P \\
M_{i}
\end{array}\right)
$$

$B_{i}$ can be understood asgenerated by $(X+1)$-SET: $\left\{G_{i}\right\},\left\{J_{1}\right\},\left\{J_{2}\right\}, \cdots,\left\{J_{X}\right\}$

3.4) $K_{i}=J_{1, i}+J_{2, i}+\cdots+J_{x, i}$, Theorem 2.3 has the similar promotion.

\section{Conclusion}

Review the whole process, $\left(\begin{array}{c}N \\ M\end{array}\right)+\left(\begin{array}{c}N \\ M+1\end{array}\right)=\left(\begin{array}{c}N+1 \\ M+1\end{array}\right) \rightarrow$ Basic Shapes in [1] $\rightarrow$ More Shapes in this article.

\section{Conflicts of Interest}

The author declares no conflicts of interest regarding the publication of this paper.

\section{Acknowledgements}

The author is very grateful to Mr. HanSan Z. (Department of Electronic Information, Nanjing University) for his suggestions on this paper.

\section{References}

[1] Peng, J. (2020) Shape of Numbers and Calculation Formula of Stirling Numbers. Open Access Library Journal, 7: e6081. https://doi.org/10.4236/oalib.1106081 